\title{
Quelques Protozoaires parasites de Mammifères sauvages à Madagascar
}

\section{Avec la description de trois nouveaux Sporozoaires}

\author{
par G. UILENBERG \\ Service d'Entomologie et Protozoologie, Laboratoire Central de l'Elevage, \\ et Institut d'Elevage et de Médecine Vétérinaire des Pays tropicaux, Tananarive (Madagascar)
}

\begin{abstract}
Résumé
Descriptions d'une nouvelle espèce de Babesiidae, Achromaticus cheirogalei, chez le lémurien Cheirogaleus major, d'un nouvel Hepatozoon, $H$. hoogstraali, chez l'insectivore Hemicentetes semispinosus, et d'une nouvelle Eimeria, E. semispinosi, également chez $H$. semispinosus. Des ookystes du type E. madagascariensis ont également été trouvés chez H. semispinosus. Quelques observations sont rapportées sur la toxoplasmose chez les lémuriens, en captivité, et sur des Plasmodia de lémuriens, en particulier $P$. girardi et $P$. lemuris chez les lémuriens splénectomisés. Une liste est donnée des ectoparasites trouvés sur les $H$. semispinosus porteurs d'Hepatozoon hoogstraali.

L'existence d'Achromaticus sp. est signalée chez 3 carnivores malgaches et un cas de toxoplasmose a été observé chez le carnivore Galidictis vittata.
\end{abstract}

\section{Summary}

Descriptions of a new species of Babesiidae, Achromaticus cheirogalei, in the lemur Cheirogaleus major, of a new Hepatozoon, $H$. hoogstraali, in the insectivore Hemicentetes semispinosus, and of a new Eimeria, E. semispinosi, also in H. semispinosus. Oocysts resembling $E$. madagascariensis were also found in $H$. semispinosus. Some observations are given on toxoplasmosis in lemurs in captivity, and on Plasmodia in lemurs, especially $P$. girardi and $P$. 
lemuris in splenectomized lemurs. A list is given of the ectoparasites found on the H. semispinosus infected with Hepatozoon hoogstraali.

The existence of Achromaticus sp. in 3 malagasy carnivores is reported and one case of toxoplasmosis has been observed in the carnivore Galidictis vittata.

La faune des protozoaires parasites d'animaux endémiques de Madagascar est encore peu connue. Nous rapportons dans cette note quelques nouvelles observations, certaines concernant des espèces nouvelles, d'autres n'étant pas suffisamment approfondies pour permettre des descriptions, ou concernant des espèces déjà connues.

\section{PROTOZOAIRES DE LEMURIENS (LEMUROIDEA, PRIMATES).}

[En ce qui concerne la classification des lémuriens, nous suivons celle adoptée par Petter (1962)].

$\mathbf{1}^{\circ}$ Achromaticus cheirogalei sp. n. (Babesiidae, Sporozoa) $\left(^{*}\right)$.

Hôte : Cheirogaleus major Geoffroy, 1812 (Famille des Lemuridae).

D'assez nombreux Achromaticus sont trouvés dans les érythrocytes d'un $C h$ major, mort d'une autre cause au jardin zoologique de Tananarive, présenté au Laboratoire Central de l'Elevage le 29 septembre 1968, aux fins de diagnostic.

DESCRIPTION (fig. 1): Parasites apigmentés dans les érythrocytes (sang du cœur). Approximativement quatre parasites pour trois champs microscopiques (agrandissement $8 \times 100$ ). Colorés au Giemsa, les parasites ont l'aspect habituel de petites Bahesiidae :

a) Formes simples. Les formes annulaires prédominent; leur diamètre varie d'un peu moins de $1 \mu$, à un peu plus de $2 \mu$. Il y a également des formes elliptiques, triangulaires, en poire, et irrégulières, le plus grand diamètre ne dépassant pas environ $2,5 \mu$. La chromatine est concentrée à une extrémité du parasite, ou suit entièrement son pourtour. L'intérieur des parasites est très clair.

b) Formes de division. Division en quatre: le premier stade observé, consiste en quatre condensations de chromatine à la périphérie du parasite, en croix. Quatre parasites minuscules (environ 0,3 à $1,0 \times 0,3$ à $0,7 \mu$ ), en résultent et se dispersent dans l'érythrocyte. Une division en deux semble également se produire, quelques globules étant infestés par deux parasites de taille égale, et de rares formes géminées ayant été observées (fig. 1).

Les quatre parasites, résultant de la division, sont souvent constitués presqu'entièrement de chromatine.

* Nous avons exposé ailleurs (Uilenberg, 1967) les raisons pour lesquelles nous considérons Achromaticus comme nom générique valable pour les Babesiidae qui se multiplient habituellement par division en quatre 
Discussion: La morphologie des parasites a pu être légèrement affectée par le fait que l'hôte était mort au moment de la confection des frottis ; il était pourtant si frais que la morphologie des thrombocytes dans le sang restait normale.

Une séparation de ce parasite de certaines autres espèces d'Achromaticus est impossible sur la morphologie, et nous basons la création d'une nouvelle espèce sur le fait qu'aucune n'en est connue chez les Lemuroidea, ni à notre connaissance chez les Primates en général (nous ne parlons pas du genre Babesia, sensu stricto, connu chez des singes).
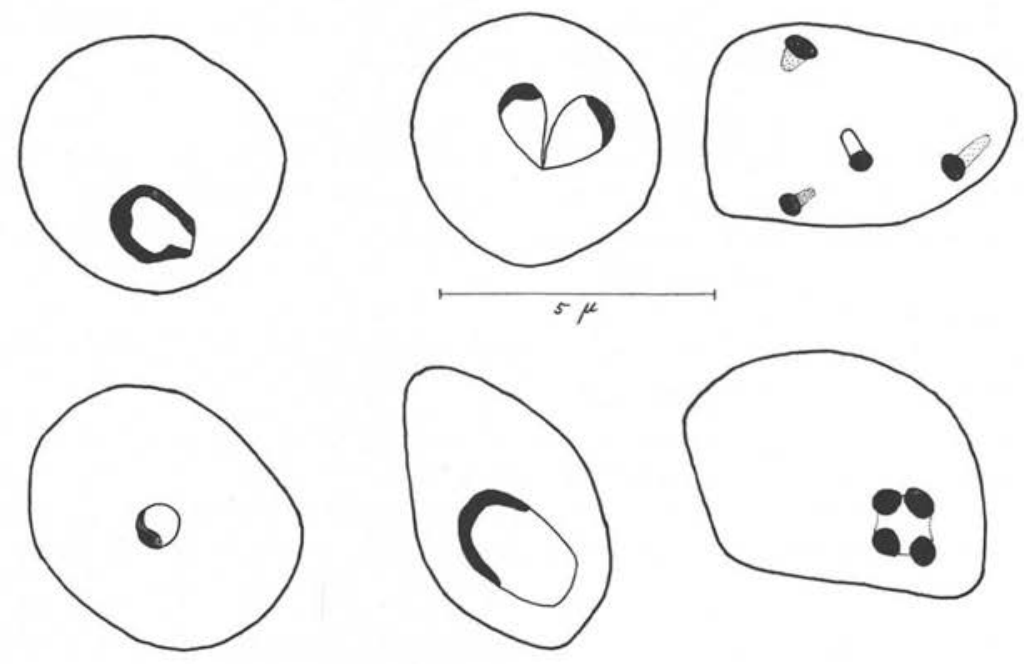

Fig. 1. - Achromaticus cheirogalei

Formes simples et formes de division

Il serait intéressant de connaître le vecteur d'Achromaticus cheirogalei; l'anımal en question ne portait aucune tique, mais deux espèces d'Ixodidae sont connues chez les Lémuriens malgaches, Haemaphysalis lemuris Hoogstraal, 1953 et Ixodes lemuris Arthur, 1957, bien qu'aucune des deux n'ait été trouvée chez un Cheirogaleus.

Des frottis contenant $A$. cheirogalei sont déposés dans la collection de l'auteur.

$2^{\circ}$ Plasmodia (Plasmodiidae, Sporozoa).

Trois espèces de ce genre ont été décrites chez les lémuriens malgaches, uniquement chez le genre Lemur, famille des Lemuridae :

a) Plasmodium girardi Bück, Coudurier et Quesnel, 1952.

Ce parasite fut mis en évidence par splénectomie d'un Lemur macaco rufus Audebert, 1800 (Bück et al., 1952). Brygoo (1963) rapporte la sortie de cette espèce sur un des deux L. macaco fulvus Geoffroy, 1812, splénectomisés par le $\mathrm{D}^{\mathrm{r}}$ J.-P. Raynaud et 
nous-même ; ce lémurien constitue donc un nouvel hôte. Garnham (1966) redécrit l'espèce d'après le matériel obtenu sur ce $L$. macaco fulvus splénectomisé ; il la place dans le sous-genre Vinckeia.

\section{Nos OBSERVATIONS :}

A) Apparition de $P$. girardi dans le sang périphérique d'un $L$. macaco macaco Linné, 1766,10 jours après sa splénectomie. Le maximum de la parasitémie (très approximativement dix érytiırocytes infestés par le champ microscopique), se situe entre le $15^{\mathrm{e}}$ et $21^{\mathrm{e}}$ jour; les parasites sont rares à partir du $31^{\circ}$ jour, mais persistent jusqu'à la fin de la période d'observation (48 jours). Aucun symptôme de maladie n'a été observé.

B) Environ $0,5 \mathrm{ml}$ de sang citraté prélevé sur ce $L . m$. macaco, 16 jours après sa splénectomie, est inoculé par voie sous-cutanée, à un L. mongoz mongoz, Linné, 1766 ; ce dernier avait été splénectomisé 23 jours auparavant sans sortie d'hématozoaires.

$P$. girardi apparaît dans le sang du $L . m$. mongoz, 13 jours après l'inoculation, augmente en nombre jusqu'à un maximum d'environ 3 à 4 érythrocytes infestés par champ microscopique, aux environs du $22^{\circ}$ jour après l'inoculation. Les parasites se font rares à partir du $27^{\circ}$ jour, mais persistent jusqu'à la fin de l'observation (32 jours). Aucun symptôme de maladie.

C) Apparition de $P$. girardi dans le sang périphérique d'un $L$. macaco fulvus, 7 jours après sa splénectomie. La parasitémie maxima (approximativement 7 érythrocytes infestés par champ microscopique), se situe entre le $11^{\circ}$ et $14^{\mathrm{e}}$ jour. Les parasites sont rares à partir du $16^{\circ}$ jour, mais persistent en faible nombre jusqu'à la fin de la période d'observation post-opératoire (81 jours). Aucun symptôme de maladie.

D) Echec de transmission de $P$. girardi, par l'injection d'environ $0,5 \mathrm{ml}$ de sang citraté, prélevé sur ce $L$. macaco fulvus, 77 jours après sa splénectomie, à un L. catta, Linné, 1758 ; ce dernier avait été splénectomisé 62 jours auparavant, sans sortie d'hématozoaires. La période d'observation après l'inoculation est de 23 jours.

Ajoutons que Brygoo (1963) rapporte une période latente après la splénectomie d'environ huit jours et une parasitémie maxima entre le $12^{\circ}$ et $25^{\circ}$ jour.

Une redescription de la morphologie de $P$. girardi sera donnée ultérieurement en collaboration avec le Professeur P.C.C. Garnham, aussi nous n'en donnons pas de détails ici.

b) Plasmodium foleyi Bück, Courdurier et Quesnel, 1952.

Cette espèce fut mise en évidence après la splénectomie du même $L$. macaco rufus qui avait sorti $P$. girardi (in Bück et al., 1952). Le parasite n'a plus été observé depuis. Huff et Hoogstraal (1963) mettent sa valeur en doute, mais une de leurs objections, à savoir que les deux espèces $(P$. girardi et $P$. foleyi) sont apparues sur le même animal à intervalle rapproché, n'est pas convaincante (voir nos observation sur $P$. lemuris ci-dessous). Garnham (1966), considérant que les dessins de Bück et al. (1952) ne montrent pas de schizontes, pense qu'il s'agisse plutôt d'une espèce d'Hepatocystis que de Plasmodium. Il nous semble actuellement impossible de se prononcer 
sur la valeur et la classification éventuelle de $P$. foleyi, en l'absence d'autres observations. La possibilité que $P$. foleyi et $P$. lemuris constituent une même espèce n'est peut-être pas entièrement à écarter.

c) Plasmodium lemuris Huff et Hoogstraal, 1963.

L'espèce a été trouvée dans le sang d'un Lemur macaco collaris Geoffroy, 1812 (Huff et Hoogstraal, 1963). Garnham (1966) la place dans le sous-genre Vinckeia, et n'écarte pas la possibilité qu'il s'agisse d'un synonyme de $P$. girardi, mais nos observations montrent qu'il n'en est rien, et que c'est bien une espèce différente.

\section{NOS OBSERVATIONS :}

A) Apparition de $P$. lemuris, dans le sang périphérique d'un L. macaco fulvus, 21 jours après sa splénectomie. (Il s'agit de l'animal qui s'était montré positif pour $P$. girardi à partir de 7 jours après l'opération, voir paragraphe $\mathrm{C}$ sous $P$. girardi).

Les $P$. lemuris restent toujours rares, et sont observés avec certitude pour la dernière fois au $57^{\circ}$ jour après la splénectomie. (La période totale d'observation post-opératoire est de 81 jours). Aucun symptôme de maladie.

B) Environ $0,5 \mathrm{ml}$ de sang citraté prélevé sur ce L. macaco fulvus, 52 jours après sa splénectomie. est inoculé par voie sous-cutanée à un $L$. mongoz mongoz splénectomisé depuis 170 jours. (Il s'agit du $L$. m. mongoz inoculé auparavant avec $P$. girardi, voir paragraphe $\mathrm{B}$ sous $P$. girardi. Le sang de cet animal a été examiné régulièrement de nouveau à partir du $107^{\circ}$ jour après sa splénectomie, sans montrer d'hématozoaires).

$P$. lemuris est observé dans le sang du L. m. mongoz 32 jours après l'inoculation, pendant un jour seulement, et en faible nombre. L'observation cesse 39 jours après l'injection. Aucun symptôme de maladie.

Une redescription de la morphologie de $P$. lemuris sera faite ultérieurement en collaboration avec le Professeur P.C.C. Garnham, les détails n'en sont donc pas donnés ici.

\section{Autre observation de Plasmodiidae:}

Rares parasites pigmentés dans les érythrocytes d'un Avahi laniger (Gmelin, 1788) (Famille des Indridae), présenté pour autopsie au laboratoire en 1966. Malheureusement, il n'y avait que quelques trophozoïtes, contractés après la mort, ce qui a empêché une étude plus approfondie, car c'est le premier enregistrement d'une espèce de Plasmodiidae chez les Indridae.

\section{Conclusions :}

P. girardi existe à l'état naturel chez L. macaca macaco, outre les premiers hôtes rapportés, L. macaco rufus et $L$. macaco fulvus.

$P$. girardi infecte expérimentalement $L$. mongoz mongoz.

Il n'a pas été possible d'infecter $L$. catta avec $P$. girardi (l'observation a tout au moins été négative pendant 23 jours après l'injection). Notons également que la splé- 
nectomie de deux $L$. catta n'a fait sortir aucun parasite sanguin pendant des périodes d'observation post-opératoire de 20 à 69 jours ; il s'agit toutefois d'animaux ayant vécu en captivité pendant des périodes inconnues.

$P$. lemuris existe à l'état naturel chez $L$. macaco fulvus, outre le premier hôte signalé, L. macaco collaris.

$P$. lemuris infecte expérimentalement L. mongoz mongoz.

Une espèce de Plasmodiidae existe chez la famille des Indridae (Avahi laniger).

$3^{\circ}$ Toxoplasma gondii Nicolle et Manceaux, 1908 (Toxoplasmatidae, incertae sedis).

Ce parasite a jusqu'ici été enregistré chez deux espèces de lémuriens malgaches : Lemur catta (par Sureau et al., 1962, Uilenberg et Ribot, 1965 et Itakura et Nigi, 1968) et Varecia variegata Kerr, 1792 (1), également de la famille des Lemuridae (par Uilenberg et Ribot, 1965).

Depuis nous avons pu faire les observations suivantes (toutes sur des lémuriens du jardin zoologique de Tananarive) :

Quatre cas chez des L. catta $(1965,1966$ et 1968).

Un cas chez un $V$. variegata (1968).

Un cas chez un Propithecus verreauxi verreauxi Grandidier, 1867, de la famille des Indridae, nouvel hôte pour ce parasite.

Dans tous les cas, comme dans ceux relatés antérieurement, la toxoplasmose a revêtu une forme aiguë, à évolution rapide, aboutissant à la mort, un à quelques jours après le début de la maladie clinique.

A l'autopsie des animaux, on observait chez quatre des six animaux un foie dégénéré, jaune, accompagné, dans un cas, d'ictère généralisé ; deux animaux avaient une pleuro-pneumonie, deux autres une congestion puimonaire ; une entérite fut remarquée sur deux des lémuriens, tandis qu'un animal a présenté une splénomégalie, un autre un hydropéricarde.

Les toxoplasmes furent dans tous les cas trouvés dans le poumon, le foie et la rate et dans cinq cas également dans le rein. Les frottis de sang en contenaient dans trois cas : toxoplasmes libres entre les cellules sanguines ou encore contenues dans des leucocytes, mais jamais dans les érythrocytes. Le poumon semble être l'organe qui contient en moyenne le plus de toxoplasmes. (Nous n'avons pas pu examiner les cerveaux, le squelette devant rester intact pour des études zoologiques).

\section{Conclusions :}

La sensibilité des lémuriens à la toxoplasmose semble exceptionnellement grande. et cette maladie occupe une place importante parmi les causes de leur mortalité (surtout des $L$. catta) au jardin zoologique.

(1) Petter (1962) écrit Varecia variegatus. 


\section{PROTOZOAIRES D'INSECTIVORES (TENRECIDAE, INSECTIVORA).}

$1^{\circ}$ Hepatozoon hoogstraali sp. n. (Haemogregarinidae, Sporozoa).

Hote: Hemicentetes semispinosus (Cuvier, 1798).

Le sang périphérique de 69 spécimens de cette espèce a été examiné. Tous ont été capturés à Amparafara et Périnet (versant est des Hauts Plateaux, à mi-chemin environ entre Tananarive et la côte est), 59 d'entre eux en mai 1968 par H. Hoogstraal, G. Randrianasolo et nous-même, les 10 autres en novembre 1968 par nous-même. Les parasites furent trouvés dans le sang de 13 d'entre eux (11 du lot de mai, 2 du lot de novembre), soit de $20 \%$ environ.

Description (Fig. 2): Gamétocytes dans les érythrocytes.
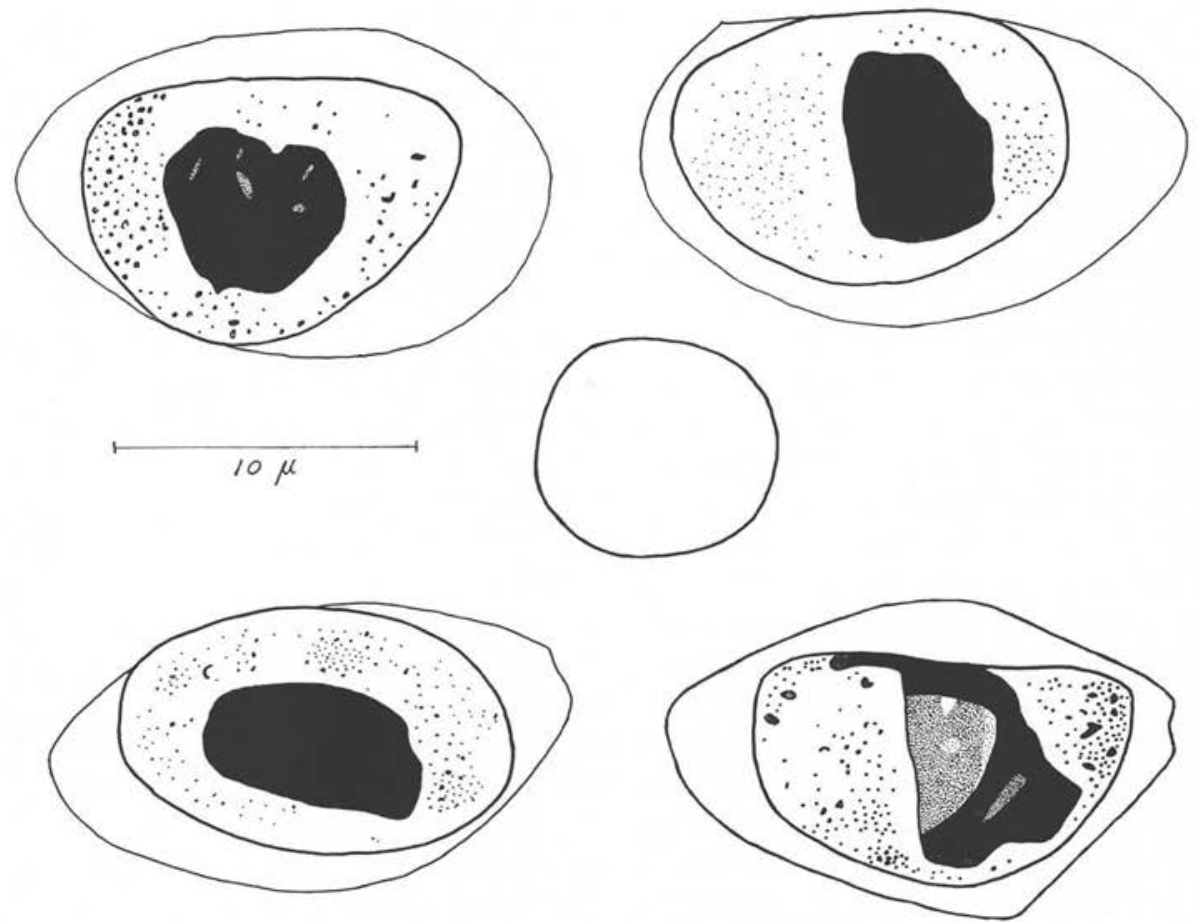

FIG. 2. - Hepatozoon hoogstraali

Au milieu un érythrocyte normal

Ces derniers sont décolorés et agrandis, et il r'en reste le plus souvent qu'une membrane transparente autour du parasite (coloration de Giemsa). Les dimensions des parasites sont d'environ 11,8 à $13,2 \mu$ sur 6,6 à $10,4 \mu$, en moyenne de $12,5 \times 8,6$. La forme est en général ovale, avec des variations allant de formes « en haricot» à 
ovoïdes. Le noyau est le plus souvent situé au centre du parasite; il est grand, de 6,6 à $9,0 \times 3,5$ à $6,3 \mu$ environ, en moyenne de $7,2 \times 5,0$; son grand axe par rapport à celui du parasite est variable, de transversal à parallèle. La structure du noyau est massive, parfois on distingue cependant des zones claires à l'intérieur. Le cytoplasma du parasite est granuleux, mais pratiquement incolore entre les granules.

L'intensité de la parasitémie variait d'un seul gamétocyte par frottis à environ un pour 3 champs microscopiques. La grande majorité des parasites est concentrée dans la queue du frottis.

Discussion : Il n'a pas été possible de trouver des stades de multiplication. Des calques et frottis de foie, de rate, de rein et de poumon, faits sur la plupart des animaux, ont donné des résultats négatifs (mis à part des gamétocytes dans les poumons, identiques à ceux rencontrés dans le sang périphérique); de même pour les coupes de poumon faites sur l'animal le plus infesté et des frottis de cortex cérébral faits sur un autre spécimen ayant une infestation sanguine assez importante. Il est possible que la schizogonie se fasse dans un autre organe (comme le pancréas, auquel nous n'avions pas pensé jusqu'à la lecture de la publication par d'Utra et al., 1916, voir ci-dessous).

En l'absence de toute connaissance sur le cycle chez le mammifère, c'est uniquement par la morphologie des formes sanguines qu'il nous semble justifié d'inclure ce parasite provisoirement dans le genre Hepatozoon. La morphologie générale se rapproche beaucoup de celle d'Hepatozoon didelphydis (d'Utra, Silva et Arantes, 1916), dont les gamétocytes furent trouvés dans les érythrocytes d'un marsupial sudaméricain (Didelphis aurita), et la schizogonie dans le pancréas. $H$. hoogstraali ressemble également beaucoup à une espèce non nommée, trouvée dans les érythrocytes d'un marsupial australien, Petaurus sp. (Mackerras, 1959), tandis que le Professeur P.C.C. Garnham (correspondance) considère sa morphologie comme étant proche d'une espèce non décrite, trouvée chez un autre marsupial sudaméricain (Metachirops opossum), mentionnée par Garnham et Lewis (1958).

C'est à notre connaissance la première fois qu'un Hepatozoon est trouvé chez un insectivore. L'isolement primitif de Madagascar du reste du monde, et le fait que les Tenrecidae (famille endémique) paraissent devoir être placées à la base de l'ordre des insectivores et n'ont survécu que grâce à leur isolement (Grassé, 1955), rendent particulièrement intéressante cette ressemblance morphologique avec des parasites de marsupiaux. Il serait toutefois prématuré de tirer trop de conclusions de la morphologie des stades érythrocytaires en l'absence de toute connaissance sur le cycle biologique.

La transmission est également inconnue. Notons seulement les ectoparasites récoltés sur les deux lots d'Hemicentetes semispinosus:

a) Tiques (Ixodidae) :

Haemaphysalis elongata Neumann, 1897.

Ixodes lunatus Neumann, 1907.

(Il est par ailleurs, connu que les espèces suivantes parasitent également $H$. semispinosus : Haemaphysalis simplex Neumann, 1897. 
Haemaphysalis subelongata Hoogstraal, 1953.

Haemaphysalis tiptoni Hoogstraal, 1953).

b) Autres Acarina :

Psylloglyphus uilenbergi Fain, 1966 (Fam. Saproglyphidae), parasitant des puces de l'espèce Synopsyllus fonquerniei Wagner et Roubaud, 1932, récoltées sur les H. semispinosus.

c) Puces (Siphonaptera) :

Paractenopsyllus pauliani Lumaret, 1962 (déterminé par J.-M. Klein).

Paractenopsyllus petiti Klein, 1965.

Synopsyllus fonquerniei Wagner et Roubaud, 1932.

(De plus, les espèces suivantes, non endémiques à Madagascar, et qui ne sauraient donc pas être considérées comme vecteurs normaux de $H$. hoogstraali :

Ctenocephalides felis strongylus Jordan, 1925.

Pulex irritans Linné, 1758.

Xenopsylla cheopis [Rothschild, 1903]).

Il est évidemment également possible que le vecteur est un diptère piqueur. Notons que Grjebine (1966, p. 461) signale des sporozoïtes d'hémogrégarines chez des femelles du moustique Anopheles funestus Giles, 1900 (espèce non endémique d'ailleurs).

Nous dédions cette nouvelle espèce au $\mathrm{D}^{r} \mathrm{H}$. Hoogstraal pour l'aide et la stimulation qu'il nous a inlassablement apportées dans l'étude des parasites, tant de Madagascar que d'autres pays.

2. Eimeria semispinosi sp. n. (Eimeriidae, Sporozoa).

HoTe: Hemicentetes semispinosus.

Des ookystes de cette nouvelle espèce furent trouvés dans les fèces de 4 sur 10 H. semispinosus, capturés à Amparafara en novembre 1968 par nous-même. L'infestation était faible sur deux d'entre eux, très importantes sur les deux autres.

DESCRIPTION (Fig. 3) :

Ookystes non sporulés: Paroi rugueuse et granuleuse, épaisse, de couleui jaunemarron ; elle donne parfois l'impression d'être composće de deux ou plusieurs couches irrégulières. Forme de l'ookyste ellipsoïde à ovoïde. Il y a souvent un amincissement de la paroi au pôle le plus pointu. L'intérieur est entièrement rempli par le sporonte (ce qui empêche d'observer la présence éventuelle d'un granule polaire).

Dimensions (mensurations sur 50 ookystes pris au hasard) : 16,4 à 21,0 $\times 11,8$ à $16,8 \mu$, en moyenne $19,2 \pm 0,17 \times 14,3 \pm 0,13 \mu$. Diamètre de la paroi environ $1,0 \mu$, pouvant dépasser $1,5 \mu$ au pôle le plus arrondi. Indice longueur/largeur 1,34 .

La sporulation a été réalisée en couche mince. dans du bichromate de potassium à $2 \%$, à une température d'environ $25^{\circ} \mathrm{C}$. La sporonte commence à se contracter 
après 2 jours. Après 5 jours (mais non après 4) les premiers sporoblastes apparaissent, dans plus de $50 \%$ des ookystes. Les premiers sporozoïtes (encore rares) sont trouvés après six jours, presque tous les ookystes sont sporulés le lendemain.

Ookystes sporulés: Très rarement présence d'un granule polaire. Absence de corps résiduel de l'ookyste. Deux sporozoïtes par sporokyste, quatre sporokystes par ookyste. Paroi du sporokyste mince. Présence d'un corps de Stieda. Présence d'un corps résiduel du sporokyste, peu important, granuleux, de forme irrégulière. Sporozoïtes sans granules

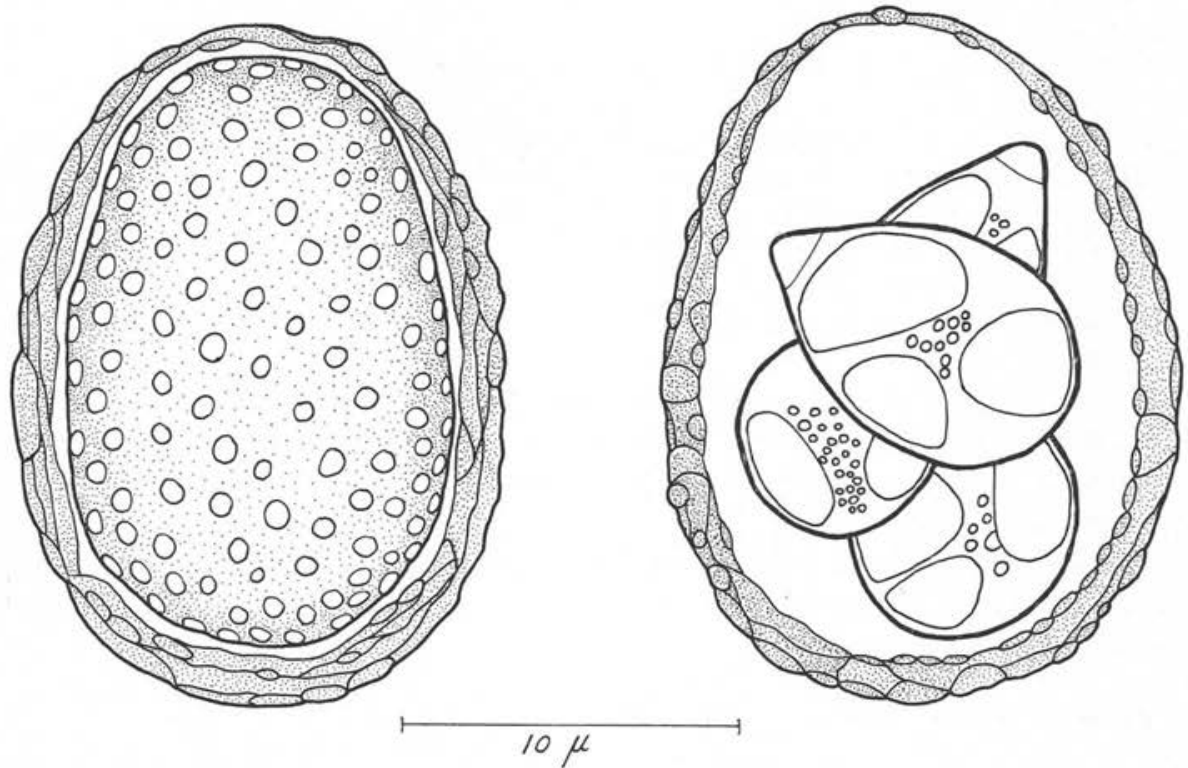

FIG. 3. - Eimeria semispinosi

Ookyste non sporulé et ookyste sporulé

réfringents ; ils semblent souvent situés en biais dans le sporokyste et être pliés en deux. Dimensions des sporokystes de 9,0 à $11,1 \times 6,3$ à $7,3 \mu$, en moyenne $10,0 \times 6,6$ (15 sporokystes mesurés).

Localisation et multiplication dans le corps: Inconnues.

Discussion: Eimeria semispinosi diffère nettement par ses dimensions, la forme de l'ookyste et d'autres caractères des deux autres espèces connues chez les Tenrecidae : E. setosi Uilenberg, 1967 et E. madagascariensis Uilenberg, 1967. La structure de la paroi se rapproche de celle d'E. setosi, mais elle est encore plus granuleuse que celle de la dérnièré.

$3^{\circ}$ Eimeria madagascariensis Uilenberg, 1967.

Les deux Hémicentetes semispinosus les plus infestés d'E. semispinosi avaient en plus d'assez nombreux ookystes du type $E$. madagascariensis dans les fèces. 
Les dimensions de ces ookystes étaient en moyenne de $16,2 \times 14,8 \mu$ (celles d' $E$. madagascariensis de Setifer setosus (Tenrecidae) de 16,3 $\times 15,4 \mu$, et celles des ookystes du type E. madagascariensis de Tenrec ecaudatus (Tenrecidae) de 17,0 × 16,3 u (Uilenberg, 1967). La morphologie des ookystes sporulés et non sporulés était identique à celle d'E. madagascariensis, à la différence près que nous n'avons pas observé de granule polaire avant la sporulation chez les ookystes d' $H$. semispinosus, tandis qu'une certaine proportion en présentait après la sporulation. Mis à sporuler dans les mêmes conditions, les premiers ookystes ayant des sporoblastes furent remarqués trois jours plus tard (très rares), le lendemain pratiquement tous présentaient des sporoblastes, après cinq jours quelques-uns étaient sporulés, pratiquement tous le lendemain.

Discussion: Trois membres de la famille des Tenrecidae sont parasités par des coccidies ayant des ookystes du type E. madagascariensis, sans qu'il soit certain s'il s'agisse d'une même espèce ou d'espèces morphologiquement très proches, mais spécifiques quant à l'hôte.

\section{PROTOZOAIRES DE CARNIVORES (VIVERRIDAE, CARNIVORA).}

\section{$1^{\circ}$ Achromaticus sp. (Babesiidae, Sporozoa).}

Nous avons pu observer en 1968 chez deux Galidictis vittata, une Fossa fossa et une Galidia elegans des protozoaires intraérythrocytaires ayant la morphologie du genre Achromaticus. Ces animaux, morts au jardin zoologique de Tananarive, ont été amenés au laboratoire aux fins de diagnostic. Etant donné que les frottis de sang ont été confectionnés si longtemps après la mort que la morphologie des parasites était altérée, nous nous bornons à en indiquer l'existence sans pouvoir donner une description détaillée.

Il s'agit d'une petite espèce ; les formes rondes les plus grandes ne dépassent pas $2 \mu$. Il existe par ailleurs de nombreuses formes très allongées et minces, jusqu'à plus de $3 \mu$ de longueur. Les formes en croix sont très rares sur nos frottis.

Le $\mathrm{D}^{\mathrm{r}} \mathrm{H}$. Hoogstraal du Caire nous a signalé (communication personnelle) qu'il a trouvé en 1948 des Babesiidae de morphologie comparable chez une Galidia elegans et une Hemigalidia olivacea, capturées dans le Sud de Madagascar; il a également observé la division en quatre.

Discussion : Il n'est pour l'instant pas possible de savoir s'il s'agit chez ces quatre représentants de Viveriidae d'une même espèce d'Achromaticus ou non.

La tique vectrice se trouve vraisemblablement parmi les Haemaphysalis endémiques à Madagascar, dont les espèces suivantes sont spécifiques de carnivores:

H. eupleres Hoogstraal, Kohls et Trapido, 1965.

H. fossae Hoogstraal, 1953.

H. obtusa Dönitz, 1910.

$2^{\circ}$ Toxoplasma gondii Nicolle et Manceaux, 1908 (Toxoplasmatidae, incertae sedis).

De très nombreux $T$. gondii sont trouvés sur les calques de poumon d'une Galidictis vittata, morte d'une maladie aiguë au jardin zoologique de Tananarive, le 7 février 
1968, et apporté au laboratoire de diagnostics. C'est le premier cas chez un carnivore sauvage à Madagascar.

\section{Bibliographie}

BRYGoo (E.-R.), 1963. - Nouvelle observation de Plasmodium girardi. Rapport sur le fonctionnement de l'Institut Pasteur de Madagascar en 1962, Tananarive, Imprimerie Nationale. Pages 267-268.

Buck (G.), Courdurier (J.) et Quesnel (J.-J.), 1952. - Sur deux nouveaux Plasmodium observés chez un lémurien de Madagascar splénectomisé. Arch. Inst. Pasteur, Algér., $30: 240-243$.

D'utra (O.), Silva et Arantes (J.-B.), 1916. - Sobre uma hemogregarina da gambá. Haemogregarina didelphydis n. sp Mem. Inst. Osw. Cruz, 8: 61-63.

Garnham (P. C. C.), 1966. - Malaria parasites and other Haemosporidia. Blackwell Scientific Publications, Oxford. 1114 pages.

Garnham (P. C. C.) et Lewis (D.-J.), 1958. - Some parasites from British Honduras. Trans. R. Soc. trop. Méd. Hyg., 52 : 295-296.

Grasse (P.-P.), 1955. - Mammifères. Les ordres: Anotamie, Ethologie, Systématique. Traité de Zoologie, Tome XVII, fasc. 2. Masson et $C^{i e}$, Paris. (Page 1642, par P.-P. Grassé, page 1699, par J. Viret).

GrJebine (A.), 1966. - Faune de Madagascar. XXII. Insectes, Diptères, Culicidae, Anophelinae. O.R.S.T.O.M., C.N.R.S. ; Imprimerie Lahure, Paris.

Huff (C. G.) et HoogstraAl (H.), 1963. - Plasmodium lemuris n. sp. from Lemur collaris E. Geoffroy. J. infect. Dis., 112: 233-236.

ITAKURA (Ch.) et Nigi (H.), 1968. - Histopathological observations on two spontaneous cases of toxoplasmosis in the monkey (Lemur catta). Jap. J. vet. Sci., 30:341-346.

Mackerras (M. J.), 1959. - The Haematozoa of Australian mammals. Aust. J. Zool., 7 ; 105-135.

Petter (J.-J.), 1962. - Recherches sur l'écologie et l'éthiologie des lémuriens malgaches Mém. Mus. Nat. Hist. nat., N. S., sér. A, 27: 1-146.

Sureau (P.), Raynaud (J.-P.), Lapeire (C.) et Brygoo (E.-R.), 1962. - Premier isolement de Toxoplasma gondii à Madagascar. Toxoplasmose spontanée et expérimentale du Lemur catta. Bull. Soc. Path. exot., 55: 357-362.

UILENBERG (G.), 1967. - Trois nouveaux parasites dinsectivores malgaches: Achromaticus brygooi sp. n. (Sporozoa Babesiidae), Eimeria setosi sp. n. et Eimeria madagascariensis sp. n. (Sporozoa, Eimeriidae). Ann. Parasit. hum. comp., 42 : 387-398.

Uilenberg (G.) et Ribot (J.-J.), 1965. - Note sur la toxoplasmose des lémuriens (Primates, Lemuridae). Rev. Elev. Méd. vét. Pays trop., 18: 247-248. 